OPEN

SUBJECT AREAS:

PERCEPTION

PSYCHOLOGY

OBJECT VISION

NEUROSCIENCE

Received

17 April 2013

Accepted

2 September 2013

Published

4 October 2013

Correspondence and requests for materials should be addressed to D.Y. (dyao@uestc.edu. $\mathrm{cn})$

\section{How Cognitive Plasticity Resolves the Brain's Information Processing Dilemma}

\author{
Diankun Gong' ${ }^{1}$ Weiyi Ma ${ }^{1}$, , Keith M. Kendrick' , Qingqing Hu' \& Dezhong Yao' \\ 'Key Laboratory for Neurolnformation of Ministry of Education, School of Life Science and Technology, University of Electronic \\ Science and Technology of China, Chengdu 610054, China, ${ }^{2}$ School of Foreign Languages, University of Electronic Science and \\ Technology of China, Chengdu 610054, China.
}

A key unresolved question in cognitive science is whether the brain uses asynchronous or synchronous patterns of information transmission. Using an auditory learning task combined with electrophysiological recordings, we reveal for the first time that cognitive plasticity during learning transforms an asynchronous into a synchronous transmission pattern to achieve rapid, error-free performance. We also present a new model showing how the brain may resolve its information processing and transmission dilemma.

A major hypothesis about information processing is that information is processed in a contingent sequence involving sensory processing, stimulus identification and response selection, preparation and execution stages or processes ${ }^{1}$. Therefore, any theory of cognitive plasticity must provide an explanation of how information is transmitted between these processing stages ${ }^{1-4}$. This study examines how information transmission occurs during learning-induced plasticity in brain cognitive systems, a much debated but largely unresolved issue in cognitive science ${ }^{5}$.

By examining the transmission speed of various attributes contained by a stimulus, research has explored the information transmission pattern between processing stages ${ }^{6}$. Even a stimulus as simple as a line-drawn triangle contains multiple attributes (e.g., shape, size, color). Under certain circumstances, some attributes could be transmitted to the next stage before the processing of others. Partial information transmission has been studied in a series of event-related potential (ERP) experiments using both visual and auditory channel information as partial information to be transmitted ${ }^{7-9}$. Research using reaction-time task paradigms with simple visual or sound attributes has led to two competing theories of information transmission. The first theory maintains that information transmission occurs in a synchronous pattern whereby processing is completed at each stage before progressing to the next ${ }^{1}$, while the second proposes an asynchronous transmission pattern, where information is processed simultaneously in different $\operatorname{stages}^{6,10}$ ( $\mathrm{see}^{11}$ for a review). Arguably, an asynchronous pattern of information transmission should allow faster initial response times, but lead to more errors due to lack of temporal congruence between stimulus components, whereas a synchronous pattern might initially produce slower response times but lead to greater accuracy by maintaining temporal congruence ${ }^{12}$.

Research, however, also revealed plasticity in the information transmission. De Sanctis and Sommer found that the information transmission pattern could be influenced by task requirements under experimental conditions ${ }^{13}$. For example, an asynchronous information transmission pattern might temporarily switch to a synchronous one (or vice versa) when the accuracy of reactions (or the speed of reaction) was required. In addition, the information transmission pattern is also related to the perceptual discriminability of the attributes of a stimulus ${ }^{9}$. The highly discriminable stimulus attribute tended to be transmitted earlier as partial information in both visual ${ }^{6}$ and auditory channels ${ }^{7,8}$. However, these studies only revealed a strategic effect, which merely brought a temporary trade-off performance under certain experimental conditions. It is also important to investigate how learning induces plasticity in information processing pattern and how learning-induced plasticity speaks to information processing theories.

This study used lateralized readiness potential (LRP), an established electrophysiological measure of information processing in the brain from the motor cortex, in the context of a "go", "no-go" reaction-time task ${ }^{14}$. The LRP is derived by subtracting the potential in the correct response hand hemisphere from that in the other hemisphere $^{15}$. Where the LRP component associated with stimulus processing and response preparation $\left(S-\mathrm{LRP}^{16}\right)$ is absent in "no-go" trials, and that during response execution $\left(\mathrm{LRP}-\mathrm{R}^{16}\right)$ is maintained, this indicates full processing has occurred prior to response execution and a synchronous pattern of information transmission. LRP-R can also be used to exclude movement complexity reduction induced by irrelevant factors ${ }^{17}$. If the S-LRP-no-go remains 
following learning, this indicates that stimulus processing is still ongoing during the response preparation phase and an asynchronous pattern of information transmission. Previously we have shown that when two sound attributes with different perceptual discriminability are used, asynchronous information transmission occurs, and the performance is not error-free. Here we have investigated if this transmission pattern is maintained where both error-free performance and fast response times are required. We hypothesized that extensive training to achieve this outcome would switch an asynchronous into a synchronous transmission pattern, thereby helping reconcile the two competing theories.

\section{Results}

Behavioral data. Behavioral results showed that for subjects receiving training on the auditory task, accuracy rate was sigificantly improved and by the third session was virtually error free (Fig. $1 b, F=22.51, p=.0002$ ). Reaction times were also improved (Fig. 1d, $F=7.51, p=.003$ ). Furthermore, the expected advantage of pitch over intensity discrimination observed initially (test $1: t=8.66, p=.000003$ ) also disappeared by the final test (test 3: $t=1.89, p=.085)$, showing that training had significantly reduced the difference in processing efficiency between them. By contrast, in the control group there were no significant improvements in performance across the three tests (Fig. 1b,c,d). More details see Table S1,S2,S3.

Event related potentials data. Analyses of evoked potentials revealed no significant differences in latencies or amplitudes of N1 and P2 components (indices of sensory processing ${ }^{18}$ ) or the LRP-R (response execution ${ }^{7}$ ) across the three tests in either group. Thus, learning had no effect on sensory or response execution processing (Figs. 2a,b, and Figs. 3a,b). Hackley and Miller (1995) suggested that complex movements induced greater amplitude of S-LRP than simple movements ${ }^{17}$. In this study, LRP-R served as an index to rule out the possibility that the disappearance of S-LRP-no-go in test 3 was simply due to the reduction in movement complexity caused by the training. Actually, in the experimental group the SLRP latency was significantly reduced across the three tests (Fig. 2c, $\left.F_{g o}=258.94, F_{n o-g o}=180.8, p s<.000001\right)$, suggesting improved stimulus identification and response preparation processing leading to reduced response times. The mean amplitude of S-LRP in test 1 was significantly larger for "no-go" trials than the baseline $(t=5.62$, $p=.00015)$ and smaller than "go" trials $(t=4.32, p=.001)$, confirming asynchronous information transmission processing ${ }^{6,8,9}$. However, the S-LRP amplitude on "no-go" trials decreased at test $2(t$ $=6.16, p=.00007)$ and disappeared at test $3(t=1.64, p=.13)$, revealing a switch into a synchronous processing pattern. No S-LRP a

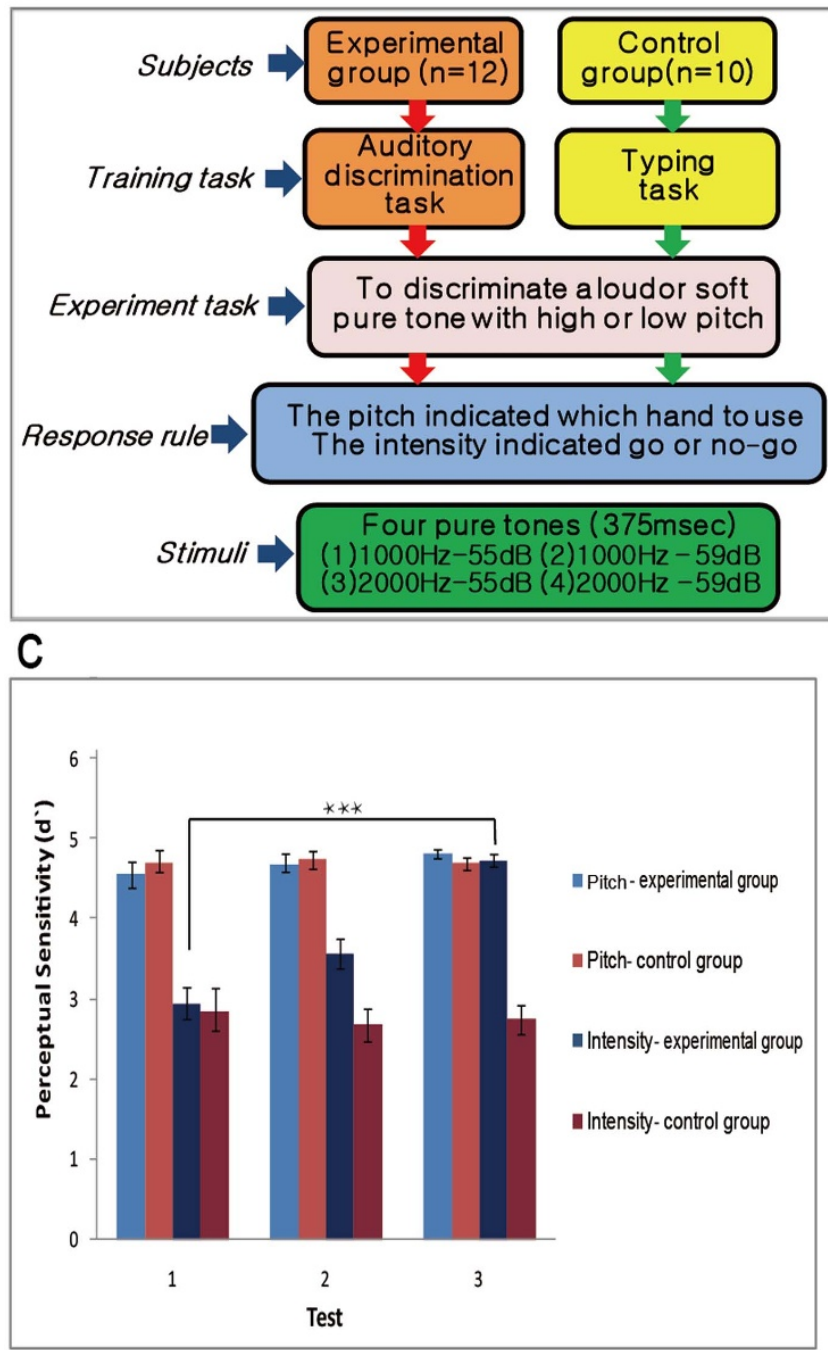

b

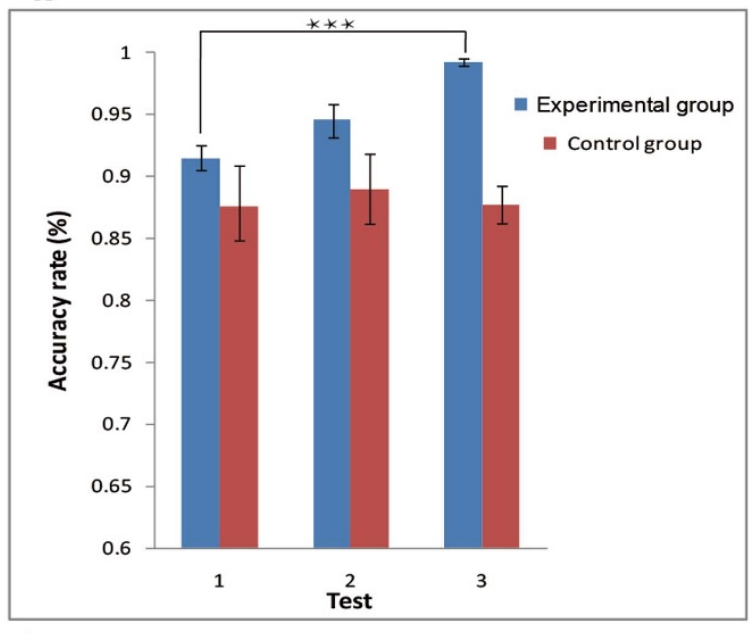

d

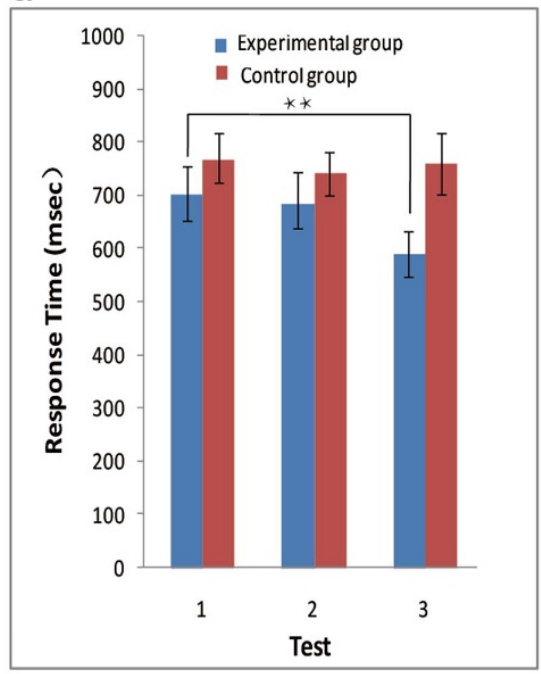

Figure 1 Experimental tasks and behavior results. (a) Experimental protocol, (b) Mean \pm Std.error accuracy, (c) pitch vs intensity sensitivity and (d) response times on the go/no-go task for experimental and control groups. ${ }^{* *} \mathrm{p}<0.001$, ${ }^{*} \mathrm{p}<0.01$ test 3 vs. test 1 . 

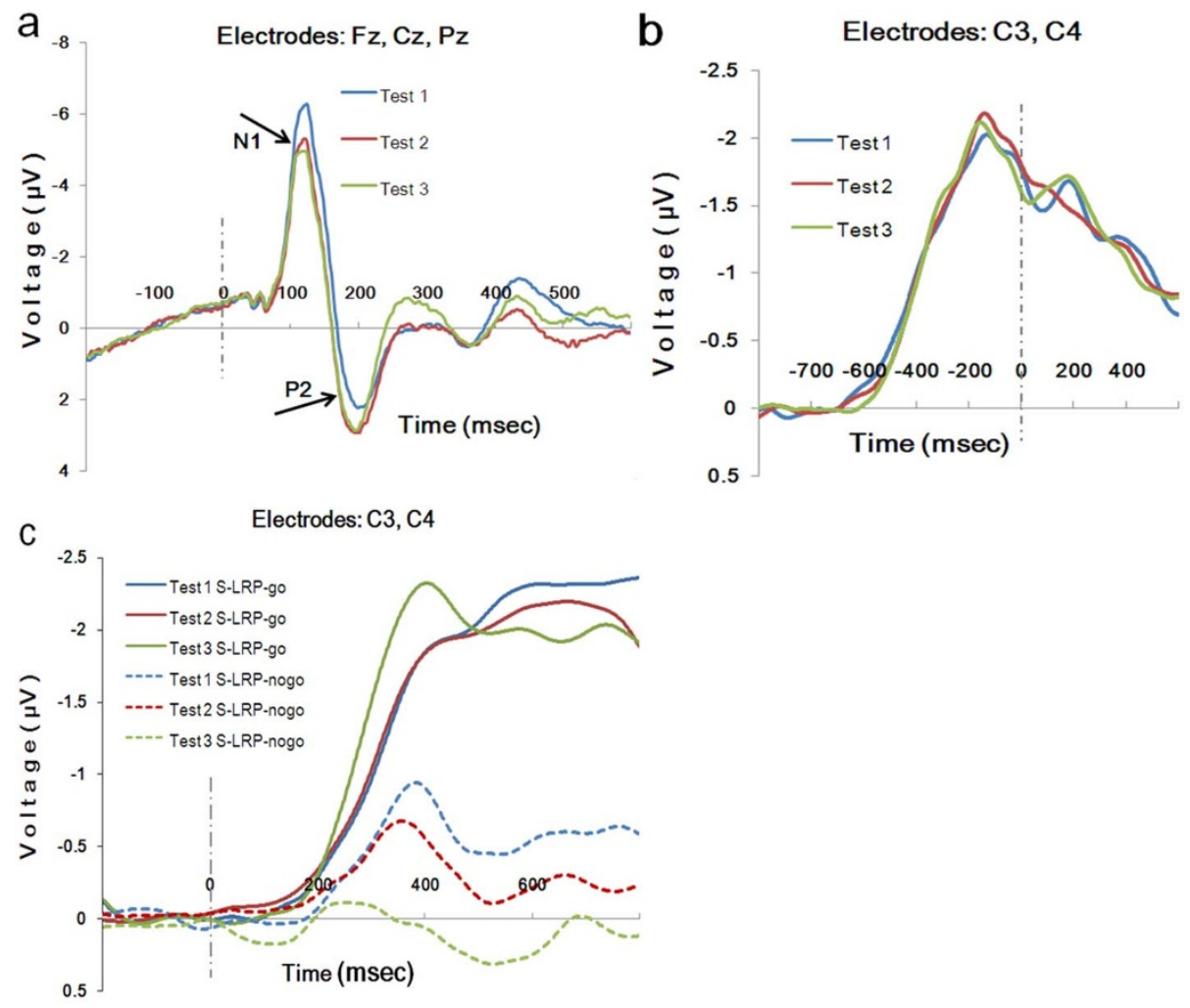

Figure $2 \mid$ Averaged evoked potentials from the experimental group showing (a) N1, P2; (b) LRP-R and (c) S-LRP latencies and amplitudes across tests.

a

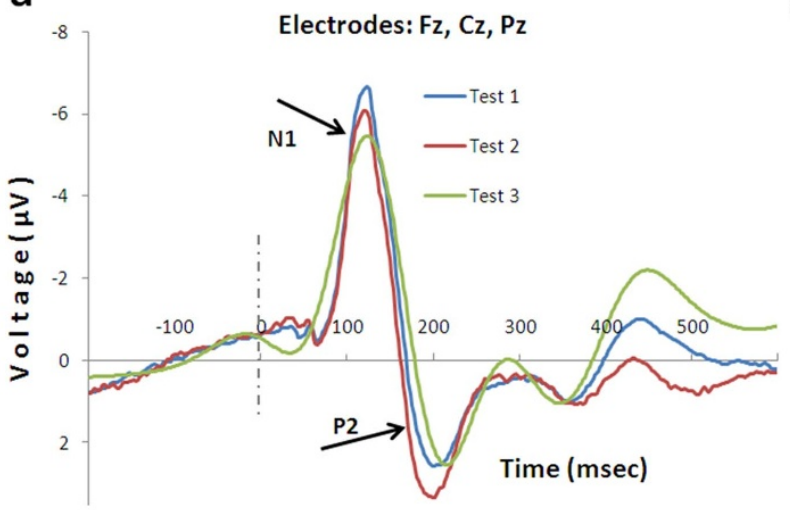

b Electrodes: C3, C4

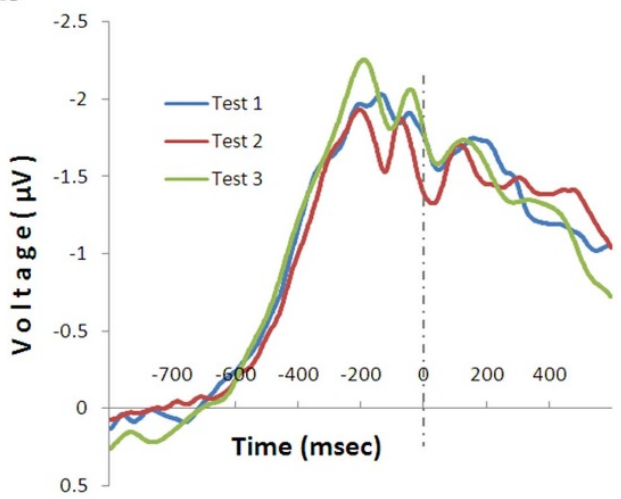

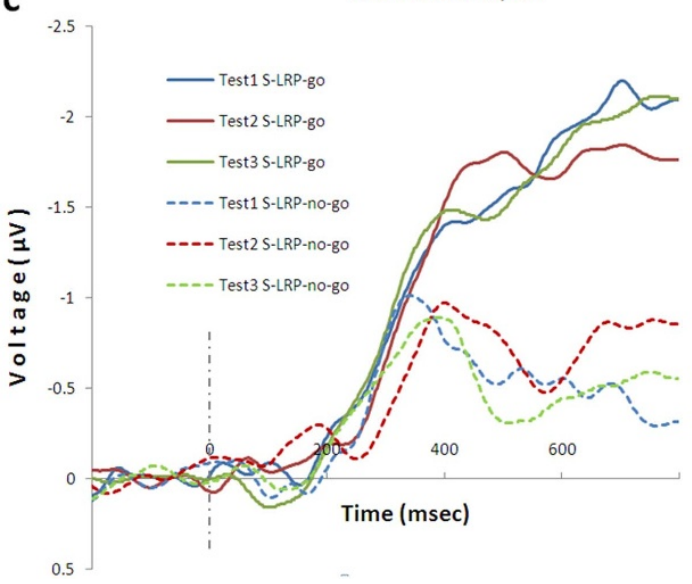

Figure 3 | Averaged evoked potentials from control group showing (a) N1, P2; (b) LRP-R and (c) S-LRP latencies and amplitudes across tests. 


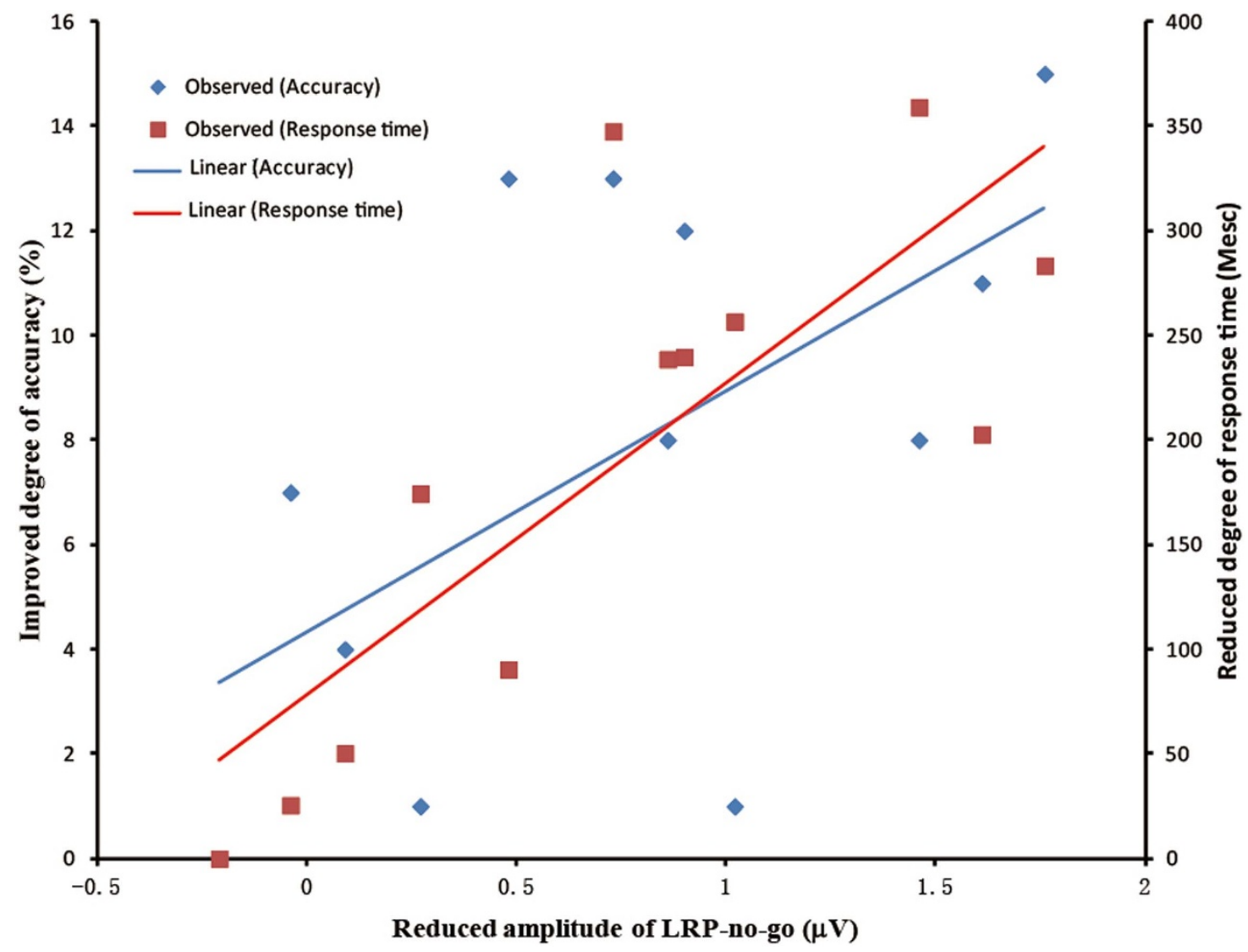

Figure $4 \mid$ Reduced amplitude of S-LRP for no-go trials was positively correlated with improved accuracy and reduced response time, supporting its relation to task performance.

\section{Test 1}

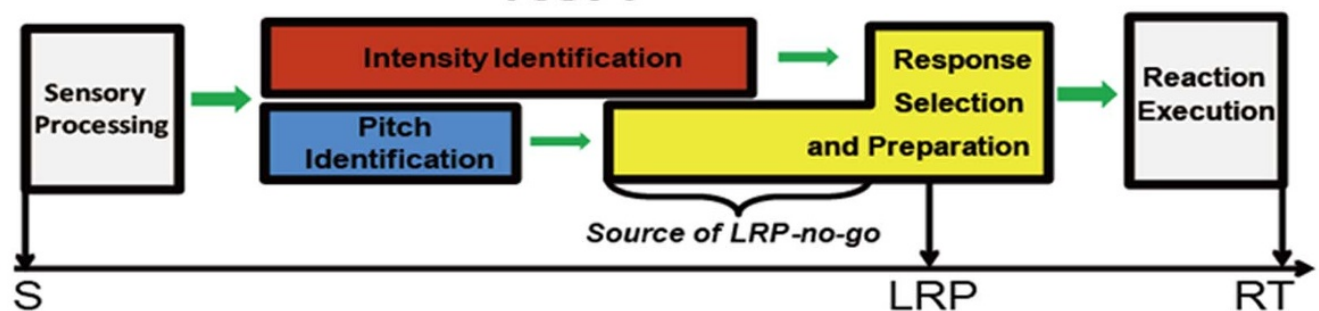

Test 2

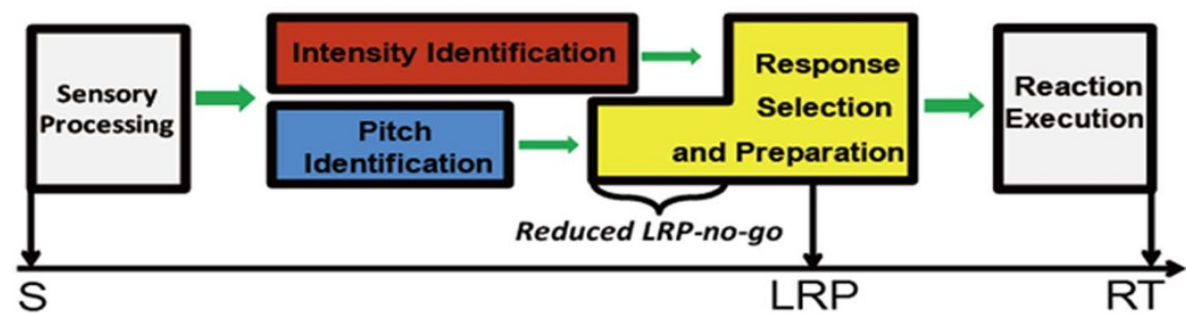

Test 3

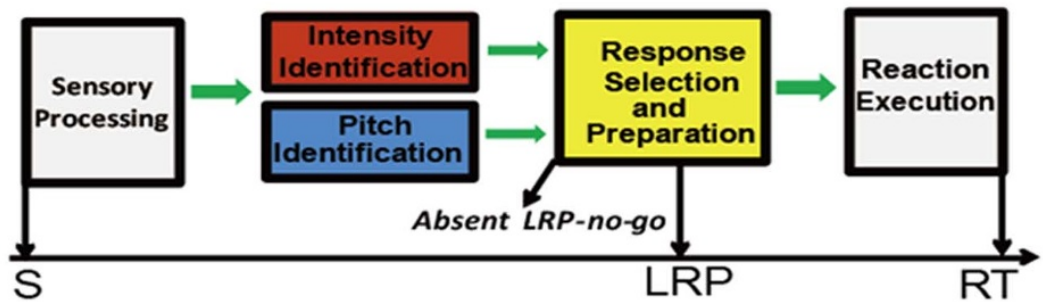

Figure $5 \mid$ Model showing how training reduces processing times for intensity and pitch until they are equivalent and the S-LRP dissappears, indicating a switch from an asynchronous to a synchronous pattern of transmission. S: Stimulus onset; RT: Reaction time.(This figure is adapted from Miller's figure 1 in $1992^{6}$.) 
changes occurred in the control group (Fig. 3c). Furthermore, by comparing participants' performance in test 1 and test 3 , we found that the reduced amplitude of S-LRP for no-go trials was positively correlated with improved accuracy $(r=0.62, p=0.03)$ and reduced response time ( $r=0.79, p=0.002)$ supporting its relationship to task performance in the experimental group (Fig. 4). More details see Table S1,S2,S3.

\section{Discussion}

From our results we propose a model summarizing how learning in this task changes an asynchronous into a synchronous pattern of information processing by improving the learners' ability to discriminate certain attributes (Fig. 5). The model proposes that pitch and intensity initially take different amounts of time to process due to variation in discrimination difficulty ${ }^{9}$. Pitch information is processed more quickly and transmitted to the response selection and preparation stage before intensity is fully processed, resulting in a large mean amplitude of S-LRP-no-go and an asynchronous transmission pattern (Test 1 in Fig. 5). As learning achieves better performance, processing time for intensity shortens disproportionately compared to pitch, resulting in a reduced mean amplitude of S-LRP-no-go and asynchronization (Test 2 in Fig. 5). Then, as learning achieves errorfree performance, processing time for intensity and pitch become equivalent and the S-LRP-no-go disappears indicating the end of continuously reduced asynchronization, and the transmission pattern has turned into a synchronous one (Test 3 in Fig. 5). For simple tasks where stimulus components have equivalent discriminability then minimal learning is required and a synchronous processing pattern will be employed from the outset.

In summary, our results help to reconcile the two conflicting theories of information processing in brain cognitive systems by demonstrating that both can occur in the same task as a function of learning. We suggest that an asynchronous processing pattern is initially employed where stimulus components in tasks have varying discriminability and processing times. The objective of cognitive plasticity during learning is to produce equivalent processing of stimulus components allowing a synchronous processing pattern to be used so rapid, error free performance is achieved.

\section{Methods}

Participants. There were 24 healthy adult subjects $(12$ female, mean $=24$ yrs, range $=21$ to $26 \mathrm{yrs}$ ), none of whom had received formal auditory training and who were randomly assigned to either experimental $(n=12)$ or control $(n=12)$ groups. Two subjects from the control group did not complete the training course and were therefore excluded. All subjects had normal hearing and normal or corrected-tonormal vision. They were reimbursed $¥ 200$. The study was done with approval from the University of Electronic Science and Technology of China Ethics Board. Informed consent was obtained from all subjects.

Stimuli and apparatus. Four pure-tone stimuli $(1000 \mathrm{~Hz}$ vs. $2000 \mathrm{~Hz} ; 55 \mathrm{~dB}$ vs. $59 \mathrm{~dB}$ ) were generated by using Adobe Audition 3.0 and rechecked by means of the PRAAT speech editing software. Tones of $375 \mathrm{msec}$ in duration were binaurally presented via headphones. The sound pressure level was double-checked by applying a digital sound level meter.

Design and procedure. The stimuli were presented in a random order in eight blocks, each consisting of 90 trials in the experimental task. On each trial, subjects were presented with a stimulus which was either a louder $(59 \mathrm{~dB})$ or softer $(55 \mathrm{~dB})$ $1000 \mathrm{~Hz}$ or $2000 \mathrm{~Hz}$ pure tone. The pitch of the tone indicated which hand to use for the response, while its intensity indicated whether a response should be performed "go" or not "no-go" (see Fig. 1a). The pitch (hand) and intensity (go/no-go) assignments were counter-balanced across subjects. The likelihood for "go" and "nogo trials" was 0.67 and 0.33 respectively. While an even likelihood of 0.5 is also effective, a higher probability for "go" trials tends to produce more consistent data ${ }^{19}$.

Subjects were seated in a dimly lit, sound attenuated and electrically shielded room, and were instructed to look at a fixation cross throughout the trials. Five hundred milliseconds before the task stimulus was presented, the fixation cross would flash to signal the upcoming critical tone, which then lasted for $375 \mathrm{msec}$. Subjects were instructed to respond quickly and accurately in the "go" trials by pressing a sequence of three keys (C, Z, and X keys in a standard key-board for left-hand responses; the comma, slash, and period keys for right-hand responses) with the index, ring, and middle fingers. This design is based on the finding that the robustness of LRPs is related to the complexity of the action required by a task ${ }^{17}$. For "no-go" trials, subjects were told to avoid making any response.

Daily training took place in a sound-attenuated laboratory. Subjects from the experimental group were trained individually for $2 \mathrm{~h}$ daily over a period of one month using the same intensity and pitch-based reaction time task. Subjects from the control group performed a completely unrelated task for $2 \mathrm{~h}$ daily (typing words on a computer keyboard as fast and as accurately as possible).

Electrophysiological (EEG) recording and data analysis. The EEG data were measured with an electrode cap of $64 \mathrm{Ag}$ - $\mathrm{AgCl}$ electrodes connected according to an extended 10-20 system and digitized with a sampling rate of $500 \mathrm{~Hz}$ (Brain Products $\mathrm{GmbH})$. The impedance for all electrodes was kept below $5 \mathrm{k} \Omega$, and all the data were band-pass filtered $(0.01-100 \mathrm{~Hz})$ online. All channels were recorded with frontal vertex (i.e., FCz) as the reference and re-referenced to "infinity" zero provided by the reference electrode standardization technique (REST) off-line ${ }^{20}$. AFz served as the ground electrode during recording. To control for eye movement artifacts, horizontal and vertical electrooculograms (EOGs) were recorded ${ }^{21}$.

Off-line EEG analysis was performed according to a standard procedure using a Brain Vision Analyzer Version 2.0.1.We computed the ERP data without offline filtering in accordance with previous recommendations ${ }^{22}$.

The $\mathrm{N} 1$ and $\mathrm{P} 2$ components of auditory evoked potentials were evaluated in terms of the average peak latency and amplitude from three middle-line electrodes (Fz, $\mathrm{Pz}$, $\mathrm{Cz}$ ). The lateralized readiness potential (LRP) was measured with the difference between the electrodes over the primary motor cortices, contra- and ipsilateral to the responding hand (i.e., $\mathrm{C} 3-\mathrm{C} 4$ and $\mathrm{C} 4-\mathrm{C} 3$ for the right- and left-hand responses, respectively). The onset latency of the stimulus-locked LRP (S-LRP) and responselocked LRP (LRP-R) were made using a Jack-Knife procedures, and the mean amplitudes were computed with a $100 \mathrm{~ms}$ time window after the onset latency ${ }^{23}$.

The $\mathrm{d}^{\prime}$ (perceptual sensitivity) is calculated according to the hit and false alarm rates $^{8}$. To calculate $\mathrm{d}^{\prime}$, one level of each stimulus dimension was arbitrarily defined as the signal and the other as noise. Specifically, for pitch analyses the pitch associated with left-hand reactions was defined as the signal and for right-hand reactions as noise. For intensity analyses, the intensity associated with the "go" response was defined as the signal and that with the "no-go" reaction as noise. Hit and false alarm rates for the pitch dimension and intensity were computed respectively and converted into $\mathrm{d}^{\prime 6}$.

Based on the standard experimental procedure and data analysis methods ${ }^{6}$, we calculated hit and false alarm rates in go trials for the pitch attribute and in both go and no-go trials for the intensity attribute. For pitch analyses, a hit was defined as a left-hand reaction to a stimulus cueing a left-hand response, regardless of whether the intensity of the tone indicated a go or a no-go response. If the subject responded with his or her left hand to the other pitch, it would constitute a false alarm. For intensity analyses, a hit was defined as a go response to an assumed go stimulus regardless of pitch, whereas a go response to any no-go stimulus constituted a false alarm." Hit and false alarm rates for the pitch and the intensity attributes were computed respectively and converted into $\mathrm{d}^{\prime}$.

1. Sternberg, S. The discovery of processing stages: Extensions of Donders' method. Acta psychol 30, 276-315 (1969).

2. Kraus, N. \& Chandrasekaran, B. Music training for the development of auditory skills. Nat Rev Neurosci 11, 599-605 (2010).

3. Mercado III, E. Neural and cognitive plasticity: from maps to minds. Psychol Bull 134, 109 (2008).

4. Anderson, J. R. Cognitive psychology and its implications. (Worth Publishers, 2009).

5. Lövdén, M., Bäckman, L., Lindenberger, U., Schaefer, S. \& Schmiedek, F. A theoretical framework for the study of adult cognitive plasticity. Psychol Bull 136, 659-676 (2010).

6. Miller, J. \& Hackley, S. A. Electrophysiological evidence for temporal overlap among contingent mental processes. J Exp Psychol Gen 121, 195-209 (1992).

7. Osman, A., Bashore, T. R., Coles, M. G., Donchin, E. \& Meyer, D. E. On the transmission of partial information: inferences from movement-related brain potentials. J Exp Psychol Human 18, 217-232 (1992).

8. Gong, D., Hu, J. \& Yao, D. Partial information can be transmitted in an auditory channel: Inferences from lateralized readiness potentials. Psychophysiology 49, 499-503 (2012).

9. Gong, D. et al. The flexibility of partial information transmission in the auditory channel: The role of perceptual discriminability. Psychophysiology 49, 1394-1400 (2012).

10. McClelland, J. L. On the time relations of mental processes: An examination of systems of processes in cascade. Psychol Rev 86, 287-330 (1979).

11. Smulders, F. T. Y., Kok, A., Kenemans, J. L. \& Bashore, T. R. The temporal selectivity of additive factor effects on the reaction process revealed in ERP component latencies. Acta psychol 90, 97-109 (1995).

12. Ho, T. et al. The optimality of sensory processing during the speed-accuracy tradeoff. J Neurosci 32, 7992-8003 (2012).

13. De Sanctis, P. \& Sommer, W. Information transmission for one-dimensional stimuli: The role of strategies. Acta psychol 131, 12-23 (2009).

14. Kenemans, J. L. \& Kähkönen, S. How human electrophysiology informs psychopharmacology: from bottom-up driven processing to top-down control. Neuropsychopharmacol 36, 26-51 (2010). 
15. Hannula, D. E., Simons, D. J. \& Cohen, N. J. Imaging implicit perception: promise and pitfalls. Nat Rev Neurosci 6, 247-255 (2005).

16. Coles, M. G. H. Modern mind-brain reading: psychophysiology, physiology, and cognition. Psychophysiology 26, 251-269 (1989).

17. Hackley, S. A. \& Miller, J. Response complexity and precue interval effects on the lateralized readiness potential. Psychophysiology 32, 230-241 (1995)

18. Hillyard, S. A. \& Kutas, M. Electrophysiology of cognitive processing. Annu Rev Psychol 34, 33-61 (1983).

19. Low, K. A. \& Miller, J. The usefulness of partial information: Effects of go probability in the choice/nogo task. Psychophysiology 36, 288-297 (1999).

20. Yao, D. A method to standardize a reference of scalp EEG recordings to a point at infinity. Physiol Meas 22, 693 (2001).

21. Gratton, G., Coles, M. G. H. \& Donchin, E. A new method for off-line removal of ocular artifact. Electroencephalogr Clin Neurophysiol 55, 468-484 (1983).

22. Woodman, G. F. A brief introduction to the use of event-related potentials in studies of perception and attention. Atten Percept Psychophys 72, 2031-2046 (2010).

23. Miller, J., Patterson, T. \& Ulrich, R. Jackknife-based method for measuring LRP onset latency differences. Psychophysiology 35, 99-115 (1998).

\section{Acknowledgements}

This work was supported by the NSFC No. 91232725 , the 863 project 2012BAI16B02, the '111' project (B12027). WM is supported by Chinese Ministry of Education Social Science
Funding (11YJC880079), National Educational Research Key Project (GPA115005), Fundamental Research Funding for Central Universities (ZYGX2010J137).

\section{Author contributions}

D.G. and D.Y. conceived and designed the experiments. D.G. and Q.H. performed the experiments. Q.H. analyzed the data. and D.G., K.K., W.M. and D.Y. wrote the main manuscript text. D.G. and W.M. prepared the figures and tables. All authors reviewed the manuscript.

\section{Additional information}

Supplementary information accompanies this paper at http://www.nature.com/ scientificreports

Competing financial interests: The authors declare no competing financial interests.

How to cite this article: Gong, D., Ma, W., Kendrick, K.M., Hu, Q. \& Yao, D. How Cognitive Plasticity Resolves the Brain's Information Processing Dilemma. Sci. Rep. 3, 2860; DOI:10.1038/srep02860 (2013)

(c) (1) (-) This work is licensed under a Creative Commons AttributionNonCommercial-ShareAlike 3.0 Unported license. To view a copy of this license, visit http://creativecommons.org/licenses/by-nc-sa/3.0 